\title{
Full energy range primary radiation damage model
}

\author{
Qigui Yang ๑ and Pär Olsson ๑* \\ KTH Royal Institute of Technology, Nuclear Engineering, Roslagstullsbacken 21, 11421 Stockholm, Sweden
}

(Received 22 February 2021; accepted 3 May 2021; published 6 July 2021)

\begin{abstract}
A full energy range primary radiation damage model is presented here. It is based on the athermal recombination corrected displacements per atom (arc-dpa) model but includes a proper treatment of the near threshold conditions for metallic materials. Both $a b$ initio (AIMD) and classical molecular dynamics (MD) simulations are used here for various metals with body-centered cubic (bcc), face-centered cubic (fcc), and hexagonal close-packed (hcp) structures to validate the model. For bcc and hcp metals, the simulation results fit very well with the model. For fcc metals, although there are slight deviations between the model and direct simulation results, it is still a clear improvement on the arc-dpa model. The deviations are due to qualitative differences in the threshold energy surfaces of fcc metals with respect to bcc and hep metals according to our classical MD simulations. We introduce the minimum threshold displacement energy (TDE) as a term in our damage model. We calculated minimum TDEs for various metal materials using AIMD. In general, the calculated minimum TDEs are in very good agreement with experimental results. Moreover, we noticed a discrepancy in the literature for fcc $\mathrm{Ni}$ and estimated the average TDE of $\mathrm{Ni}$ using both classical MD and AIMD. It was found that the average TDE of Ni should be $\sim 70 \mathrm{eV}$ based on simulation and experimental data, not the commonly used literature value of $40 \mathrm{eV}$. The most significant implications of introducing this full energy range damage model will be for estimating the effect of weak particle-matter interactions, such as for $\gamma$ - and electron-radiation-induced damage.
\end{abstract}

DOI: 10.1103/PhysRevMaterials.5.073602

\section{INTRODUCTION}

Radiation damage is a very general phenomenon that affects many scientific branches and areas of application, with examples from medicine [1], particle physics [2], applied computer science [3], space and aerospace [4], and not the least nuclear energy production, where intense neutron irradiation is in many cases life limiting for components due to radiation-induced degradation [5].

The mechanism of radiation damage production in crystalline materials can be divided into two stages based on time scale. The first one is the primary damage stage. It is the atomic collision process that takes place after highly energetic particles (electrons, neutrons, or ions) interact with atoms in a material. The actual energy transfer in the initial interaction, where the transferred energy is partitioned between electronic excitation and ionic kinetic energy, is extremely rapid and not considered as part of the damage evolution stage. The initial stage is instead defined in terms of atomic displacements. The duration of the primary damage stage is typically $<10 \mathrm{ps}$, based on various experimental and simulation studies [4]. The second stage is the defect evolution stage. This is the thermally activated process which takes place over longer

\footnotetext{
*Corresponding author: polsson@kth.se
}

Published by the American Physical Society under the terms of the Creative Commons Attribution 4.0 International license. Further distribution of this work must maintain attribution to the author(s) and the published article's title, journal citation, and DOI. Funded by Bibsam. time scales (from nanoseconds to years, depending on the conditions). Depending on the mechanism of damage production, a proper estimate of the damage rate induced by the radiation in the primary damage stage is an important part in studying radiation effects on materials.

The first step in generating such an estimate is to determine the number of defects (Frenkel pairs) produced in a single primary damage event. For each material, the most accurate way to know this is to perform experiments directly or run molecular dynamics (MD) simulations to get the average number of defects at different damage energies. However, this is often impractical due to the associated high cost and complexity, either in terms of experiment or in computation. Therefore, it is vital for many applications to have a simple but still reliable analytical model which gives a reasonable estimate of the number of produced defects. An analytical model to calculate the damage dose, in the canonical unit displacements per atom (dpa), was proposed by Kinchin and Pease (KP) [6]. The KP model calculates the damage dose by including the threshold displacement energy (TDE), denoted $E_{d}$, as the fundamental materials parameter. In the KP model, the number of defects is calculated as the kinetic energy divided by two times the TDE, after an initial sharp threshold, and up to a high-energy threshold where electronic stopping dominates. This model was later refined by Norgett et al. (NRT) [7]. Their binary collision simulations showed that $\sim 20 \%$ of the vacant lattice sites are always refilled by other atoms. Hence, they added a prefactor of 0.8 to the KP model, and they also replaced the kinetic energy by the damage energy, which includes a more realistic, physically consistent, and smooth implementation of the effect of electronic stopping for higher energies. The damage energy, denoted $T_{d}$, is thus the kinetic energy available 
to cause atomic displacements after removing the electronic ionization energy. While this NRT-dpa model is more realistic than the KP model, it still significantly overestimates the defect production in collision cascades. In fact, the actual defect production is only $\sim \frac{1}{3}$ of the NRT prediction for metallic materials, based on experiments and simulations $[4,8,9]$. Thus, a more physically realistic model is desired. Recently, an athermal recombination corrected (arc) dpa model [9] was proposed.

Compared with the KP and NRT-dpa models, the arc-dpa model provides a more accurate estimate of the number of defects for intermediate and high damage energy collision cascades, which are typically induced by neutrons in nuclear reactors [4]. However, for low-energy events, such as those from electron and $\gamma$-ray interactions, none of the current models give reasonable estimates of the number of induced defects. This is because the damage energies of the primary knock-on atom (PKA) transferred from electron and $\gamma$ rays are close to the TDE of many materials, and the effect of the sharp threshold in the KP, NRT, and arc-dpa models risk giving unphysical results. It is well known from both experiments and MD simulations [4,10-13] that each lattice direction has its own TDE. An underlying assumption in the KP, NRT, and arc-dpa models is that the TDE is a unique value in a material. This application of an averaged effect is only correct for high damage energies. The assumption is incorrect when the statistical average number of defects produced cannot be simply assumed to be 0 or 1 when $T_{d}<2 E_{d} / 0.8$. In fact, it starts to be possible to produce defects when the damage energy surpasses the minimum TDE, not the average TDE, and the probability to generate a stable defect is still not unity even when the damage energy is slightly higher than the average TDE [4,10-13]. Therefore, while the arc-dpa model is already satisfactory at high damage energies, we still need a proper model for the near TDE damage events. The importance of a proper near TDE model is evident for all applications that generate energy transfer events near the threshold. One such example is $\gamma$-ray-induced damage, which may be generated due to decay of spent nuclear fuel and can produce defects in the repository materials that have to be structurally intact over hundreds of thousands of years. According to previous models, such as KP, NRT, and arc-dpa, $\gamma$-induced damage would in most cases be strictly zero, as we will see later, because of the unphysical sharp threshold. Thus, an updated model is vital for quick but accurate estimates of the effect of $\gamma$ or electron irradiation of materials. Cosmic ray irradiation of space and terrestrial applications may also have significant fluxes of events near the TDE in critical materials.

In this paper, we propose a modification to the arc-dpa model to make it suitable for the full range of energies, including near TDE conditions. Classical MD simulations are performed to validate the modified model in the full energy range (both near TDE and for high damage energies), and we compare with available experimental data. $A b$ initio MD (AIMD) simulations are also performed to calculate the TDE of various body-centered cubic (bcc), face-centered cubic (fcc), and hexagonal close-packed (hcp) metal materials in specific lattice directions in comparison with TDE obtained from MD simulations and with experiments.

\section{METHOD}

\section{A. Analytical damage model}

The arc-dpa model is formulated as a modification on the NRT model to account for athermal recombination (recombination of defects in the primary damage stage) [9]. The number of defects $\left(N_{d}\right)$ in a collision cascade calculated by the arc-dpa model is

$$
N_{d}\left(T_{d}\right)= \begin{cases}0, & T_{d}<E_{d} \\ 1, & E_{d}<T_{d}<\frac{2 E_{d}}{0.8}, \\ \frac{0.8 T_{d}}{2 E_{d}} \xi\left(T_{d}\right), & \frac{2 E_{d}}{0.8}<T_{d}\end{cases}
$$

where $T_{d}$ is the damage energy, $E_{d}$ is the TDE of the material, and $\xi$ is

$$
\xi\left(T_{d}\right)=\frac{1-c_{\text {arcdpa }}}{\left(2 E_{d} / 0.8\right)^{b_{\text {arcdpa }}}} T_{d}^{b_{\text {ardpa }}}+c_{\text {arcdpa }},
$$

in which $b_{\text {arcdpa }}$ and $c_{\text {arcdpa }}$ are materials constants.

According to Eq. (1), $N_{d}$ is strictly zero when $T_{d}<E_{d}$; $N_{d}$ is always 1 when $T_{d}>E_{\mathrm{d}}$ and $T_{d}<2 E_{d} / 0.8$. However, $E_{d}$ is not a unique value in any given material. Here, $E_{d}$ is the average TDE over all lattice directions. This means that the TDE of each lattice direction can be either lower or higher than $E_{d}$. Therefore, $N_{d}$ should be $>0$ when $T_{d}$ is higher than the minimum TDE in a material, and $N_{d}$ should be $<1$ when $T_{d}$ is not much higher than $E_{d}$. Although this difference does not affect the $N_{d}$ of collision cascades with high damage energy (usually neutron- or ion-induced cascades), it is vital for $\gamma$ - and electron-induced collision cascades. For example, Cs-137, one of the common fission products, emits $662 \mathrm{keV}$ $\gamma$ rays during its decay via metastable Ba-137. There are two steps for $\gamma$ rays to transfer kinetic energy to atoms in materials. Firstly, the $\gamma$ rays will transfer kinetic energy to electrons due to the photoelectric effect, pair production, or Compton scattering, but for these energy ranges, Compton scattering dominates completely [14]:

$$
E_{e}=\frac{E_{\gamma}^{2}(1-\cos \theta)}{E_{\gamma}(1-\cos \theta)+m_{e} c^{2}},
$$

where $E_{e}$ is the kinetic energy transferred to the electron, $E_{\gamma}$ is the energy of the $\gamma$ ray, $\theta$ is the photon scattering angle, $m_{e}$ is the electron mass, and $c$ is the speed of light. After Compton scattering, the second step is that the scattered electrons will transfer kinetic energy to lattice atoms. The collisions between electrons and atoms need to be described by relativistic quantum mechanics because the energies of electrons are high enough to reach relativistic speed. The energy transfer between the atom and the relativistic electron can be described as follows [15]:

$$
T_{d}=\frac{2 E_{e}}{M c^{2}}\left(E_{e}+2 m_{e} c^{2}\right) \sin ^{2} \frac{\phi}{2},
$$

where we replace the kinetic energy by the damage energy $T_{d}$, given that, in the range of $10-100 \mathrm{eV}$ relevant here, electronic stopping only removes $\sim 5 \%$ of the energy. Here, $M$ is the atom mass, and $\phi$ is the electron scattering angle.

Based on Eq. (3), the $\gamma$ rays emitted from Cs-137 can transfer at maximum $\sim 477 \mathrm{keV}$ kinetic energy to electrons in the material. Relativistic electrons of such energy can ultimately 
transfer at maximum $\sim 25 \mathrm{eV}$ kinetic energy to atoms like $\mathrm{Fe}$ and $\mathrm{Cu}$ according to Eq. (4). The average TDE of $\mathrm{Fe}$ and $\mathrm{Cu}$ is $>30 \mathrm{eV}$, and such $\gamma$ rays would thus lead to no damage according to previous models, while the minimum TDE of $\mathrm{Fe}$ and $\mathrm{Cu}$ is $\sim 20 \mathrm{eV}[16]$. Hence, the $\gamma$ rays and electrons which only induce damage energies below TDE can in fact produce Frenkel pairs from scattering in lattice directions with relatively low TDEs.

Based on this, we modified the arc-dpa model by introducing the minimum TDE $E_{\mathrm{d}}^{\mathrm{min}}$ as a parameter:

$$
N_{d}\left(T_{d}\right)= \begin{cases}0, & T_{d}<E_{\mathrm{d}}^{\min } \\ \frac{0.8 T_{d}}{2 E_{d}^{\text {avr }}}, & E_{d}^{\text {min }}<T_{d}<\frac{2 E_{d}^{\text {avr }}}{0.8}, \\ \frac{0.8 T_{d}}{2 E_{d}^{\text {avr }}} \xi\left(T_{d}\right), & \frac{2 E_{d}^{\text {avr }}}{0.8}<T_{d}\end{cases}
$$

in which $E_{d}^{\mathrm{avr}}$ is the average TDE [same as $E_{d}$ in Eq. (1)]. All other parameters are the same as in the arc-dpa model. The difference between the arc-dpa model and our modification is that the modified model shows a linear relation between $N_{\mathrm{d}}$ and $T_{d}$ when the $T_{d}$ is between $E_{\mathrm{d}}^{\mathrm{min}}$ and $2 E_{d} / 0.8$. Although this adds one parameter to the model, it is a parameter that is accessible to both experiments and atomistic simulations, much more so than the average TDE.

\section{B. Classical MD simulation}

The classical MD simulations presented here consist of two parts: the near TDE simulations and the high damage energy simulations. All classical MD simulations were carried out using the code LAMMPS [17]. For the near TDE collision cascades, the simulation cell size was $12 \times 12 \times 16$ conventional lattice units. The size of the simulation cells is large enough, as has been shown earlier, for careful calculation of the TDE for Fe [13]. The simulation temperature used was $10 \mathrm{~K}$. Many evenly distributed lattice directions were sampled, respecting spherical symmetries. Atoms were assigned random initial velocities with a Gaussian distribution for the simulation temperature at the beginning of the simulation and were then equilibrated for $10 \mathrm{ps}$. After equilibration, for each lattice direction, one atom was selected and given a kinetic energy impulse in each direction. These simulations for each direction and energy were repeated three times for statistics. The number of Frenkel pairs for each kinetic energy was determined after a simulation time of 6 ps. The probability of having a Frenkel pair for each energy was then calculated by averaging the number of generated Frenkel pairs in all directions. For the high energy collision cascades, the cell size was $40 \times 41 \times 42$ lattice units, and the simulation time was $10 \mathrm{ps}$. Atoms were given a kinetic energy ranging from 0.3 to $20 \mathrm{keV}$ in the $\langle 135\rangle$ direction, which is a typical low-symmetry direction that represents well randomness for simulating collision cascades [18]. Frenkel pairs were identified using WignerSeitz analysis implemented in OVITO [19,20].

\section{AIMD simulation}

In this paper, we also determine $E_{\mathrm{d}}^{\mathrm{min}}$ in metallic materials by employing AIMD simulations. It was shown in a previous study [18] that AIMD is a powerful tool which can be applied to determine the TDE. However, due to the high complexity and computational cost, it is difficult to use

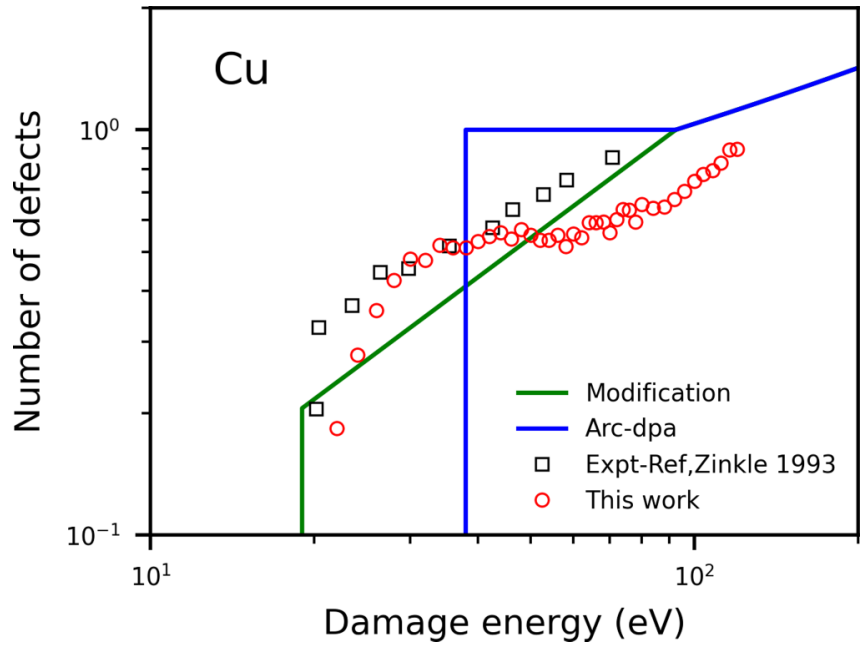

FIG. 1. Damage energy models displayed for face-centered cubic (fcc) $\mathrm{Cu}$, with comparison with experiments and classical molecular dynamics (MD) simulations. When $N_{d}<1$, it is the probability to produce a stable Frenkel pair in each damage event. For the experimental reference damage data, we name them as Zinkle 1993 [12]; the damage energy of the experimental data is determined from the recoil energy [9]; the $\mathrm{Cu}$ interatomic potential used in this work are from Ref. [25]. $E_{d}^{\min }$ and $E_{d}^{\text {avr }}$ in our modified model were calculated from classical MD simulations for self-consistency.

AIMD to search for the global $E_{\mathrm{d}}^{\mathrm{min}}$ of various materials over all lattice directions. Nevertheless, since $E_{\mathrm{d}}^{\min }$ usually exists in high-symmetry directions, we can with good confidence determine $E_{\mathrm{d}}^{\min }$ of any material by only calculating the TDE of several high-symmetry directions. Furthermore, we can minimize the number of samplings in the threshold search by using experience from classical MD simulations. All AIMD simulations were performed in the microcanonical (NVE) ensemble using the Vienna Ab initio Simulation Package (VASP) [21] with the projector augmented-wave method [22]. The generalized gradient approximation with the Perdew-BurkeErnzerhof exchange-correlation functional [23] was used for all calculations. For bcc metals, noncubic $7 \times 6 \times 6$ supercells containing 504 lattice sites were constructed. This size of supercell is large enough to sample the TDE according to previous studies [18]. For fcc and hep metals, noncubic $6 \times 5 \times 5$ supercells with 600 lattice sites were constructed. We used the standard pseudopotential for Al and semicore pseudopotentials for all other metals to have proper shortrange interactions and yield more correct TDEs [18,24]. All calculations were carried out using the $\Gamma$ point in the Brillouin zone. The search for the TDE was done by successively increasing the kinetic energy of the PKA until a stable Frenkel pair was seen after a simulation time of 2 ps, as in Ref. [18]. This minimum kinetic energy for having a stable Frenkel pair is the TDE for a particular direction. Even though the simulation time of AIMD is 4 ps shorter here than that in classical MD, no recombination of defects was observed in the classical MD simulations between 2 and 6 ps. This is because near TDE collision cascades cool down quickly due to low damage energy. Once stable Frenkel pairs are formed, just a few picoseconds are not enough for them to thermally migrate and recombine. 


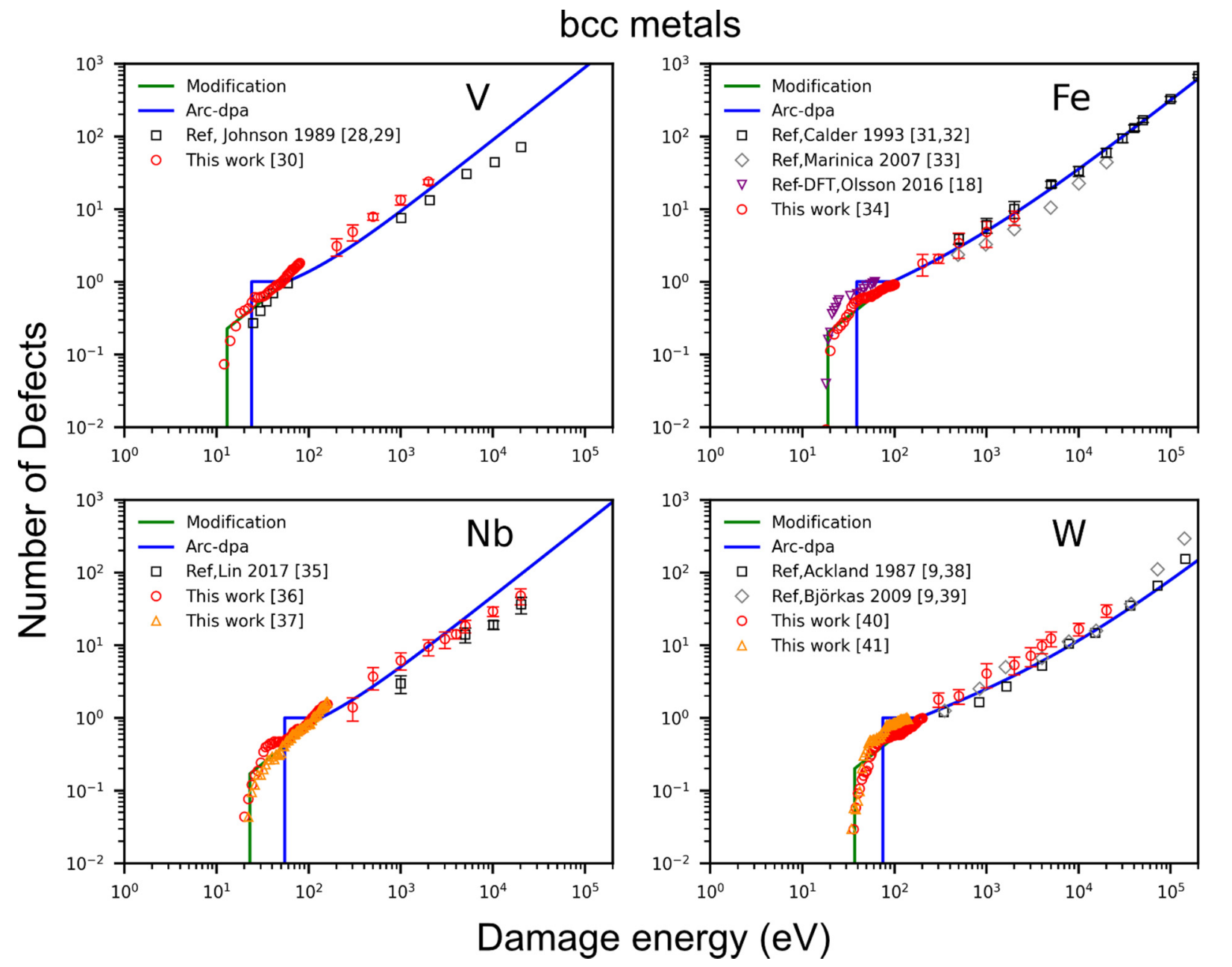

FIG. 2. Damage production in body-centered cubic (bcc) metals. Classical and ab initio molecular dynamics (MD) simulations, as well as the athermal recombination corrected displacements per atom (arc-dpa) model and our low-energy extension are compared. For our own classical MD simulations, the reference numbers are the interatomic potentials used in this work; for the reference damage data using classical MD simulations, we name them as last name of the interatomic potential developer and publication year of the potential for better comparison; for the density functional theory (DFT) reference data, we name them as last name of first author and publication year. The reference numbers for reference classical MD data contain both simulation works and potential development works (some of the potential developers also performed simulations). Reference data: V: Johnson 1989 [28,29]; Fe: Calder 1993 [31,32], Marinica 2007 [33], Olsson 2016 [18]; Nb: Lin 2017 [35]; W: Ackland 1987 [9,38]; Björkas 2009 [9,39]. Interatomic potentials used in this work: V [30], Fe [34], Nb [36,37], W [40,41]. $E_{d}^{\min }$ and $E_{d}^{\text {avr }}$ were calculated from classical MD simulations for self-consistency. The error bars correspond to standard deviation.

\section{RESULTS AND DISCUSSION}

\section{A. Near TDE damage in fcc $\mathrm{Cu}$}

In Fig. 1, we show the damage production as a function of damage energy in fcc $\mathrm{Cu}$. Copper is chosen here as a first illustration of the model because there are both experimental results and simulations to compare with. As we shall see later, the step function of the earlier models, here exemplified with the arc-dpa model, does not compare well with available data for near-threshold events. According to Fig. 1, both experimental and simulation results are better explained by the modified model than by the arc-dpa model. The modified model can provide an overall more reasonable estimation of damage production in $\mathrm{Cu}$ when the damage energy is near the TDE, even though it is very simple. However, while both the modified model and experimental results show that the increase of damage production probability is essentially linear, there is a nonlinear relationship in the MD simulation results. The reason for this will be discussed in Sec. III B.

\section{B. Defect production in bcc, fcc, and hep metals}

Figures 2-4 show the comparison between the modified model and MD simulation results for various bcc, fcc, and hcp metals, respectively. In Figs. 2 and 4, it is seen that the modified model generally shows excellent agreement with bcc and hcp metals. For fcc metals in Fig. 3, even though there are nonlinearities in the simulation results, the modified model still agrees well with simulation results. Hence, we can conclude that our modified model is general for metals and completes the arc-dpa model to cover the full energy range.

The reason for the nonlinearities in the fcc results is that the TDE surfaces of fcc metals obtained from MD simulations are qualitatively different from the other metal structures. In our 


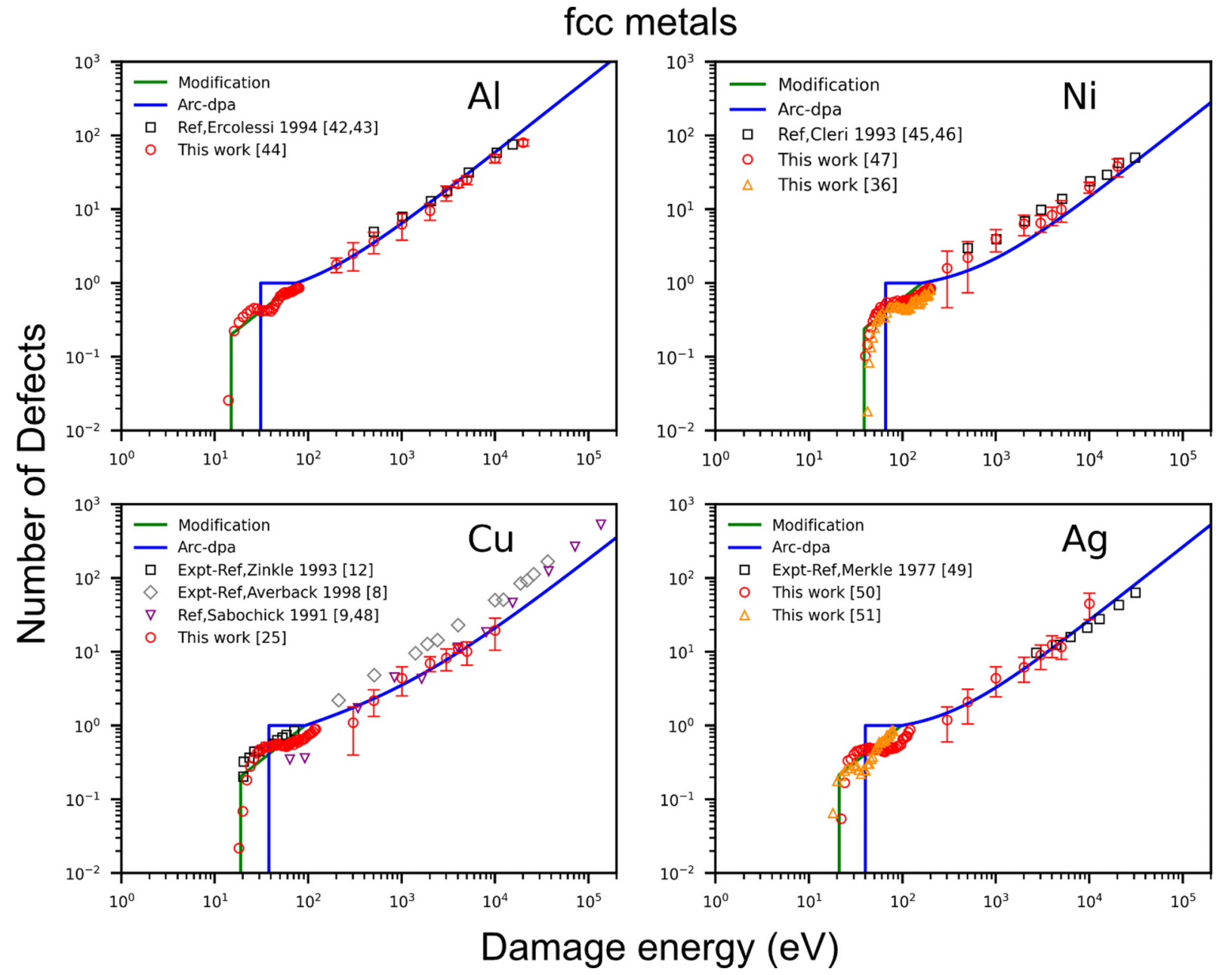

FIG. 3. Damage production in face-centered cubic (fcc) metals. Classical molecular dynamics (MD) simulations, experimental data, as well as the athermal recombination corrected displacements per atom (arc-dpa) model and our low-energy extension are compared. For our own classical MD simulations, the reference numbers are the interatomic potentials used in this work; for the reference damage data using classical MD simulations, we name them as last name of the interatomic potential developer and publication year of the potential for better comparison; for the experimental reference data, we name them as last name of first author and publication year. The reference numbers for reference classical MD data contain both simulation works and potential development works. Reference data: Al: Ercolessi 1994 [42,43]. Ni: Cleri 1993 [45,46]; Cu: Zinkle 1993 [12], Averback 1998 [8], Sabochick 1991 [9,48]; Ag: Merkel 1977 [49]. Interatomic potentials used in this work: Al [44], Ni [36,47], $\mathrm{Cu}$ [25], $\mathrm{Ag}$ [50,51]. $E_{d}^{\mathrm{min}}$ and $E_{d}^{\text {avr }}$ were calculated from classical MD simulations for self-consistency. The damage energy in the experimental data was determined from the recoil energy for $\mathrm{Cu}$ [9] and $\mathrm{Ag}$ [27]. The error bars correspond to standard deviation.

modification, it is assumed that the distribution of TDEs from minimum to maximum is uniform, meaning that the cumulative distribution function (CDF) for TDEs should increase linearly with energy. For bcc and hcp metals, this assumption is relatively accurate. Figure 5 illustrates such uniform TDE distribution for bcc Fe. It is seen that, while the increase of the CDF is not completely linear, the TDE distribution is still relatively uniform and fits well with the assumption of our modified model. For fcc metals, there are two types of TDE surfaces for explaining the kinks in the results. The first type is demonstrated in Fig. 5 for Ag. It has a similar shape with the simulation result of $\mathrm{Ag}$, meaning that the threshold energies for this metal in all directions are either very low or rather high, i.e., there are almost no directions with nearaverage level TDEs. Therefore, the number of defects will first increase rapidly with damage energy and produce defects in the low-TDE directions, then it will stop increasing until the damage energy is high enough to produce defects in high-TDE directions, opening a new regime. The second type of TDE surface is shown in Fig. 5 for $\mathrm{Cu}$ (the classical MD simulation result), where the number of defects first increase rapidly with damage energy in a similar shape with the corresponding CDF of the TDE. When the damage energy is high enough, there will on average be $>1$ defect produced in a single collision in the low-TDE directions. Hence, an abrupt increase of number of defects appears, as shown in Fig. 3 for $\mathrm{Cu}$. For the fcc metals studied here, $\mathrm{Ag}$ and $\mathrm{Al}$ have the first type of TDE surface, and $\mathrm{Cu}$ and $\mathrm{Ni}$ have the second type of TDE surface, based on classical MD simulations. The two types of TDE surfaces are also observed in experiments. Figure 3 compared the experimental TDE distributions with the simulated TDE distribution in $\mathrm{Cu}$. Experimental results in Refs. [11] and [10] have first 


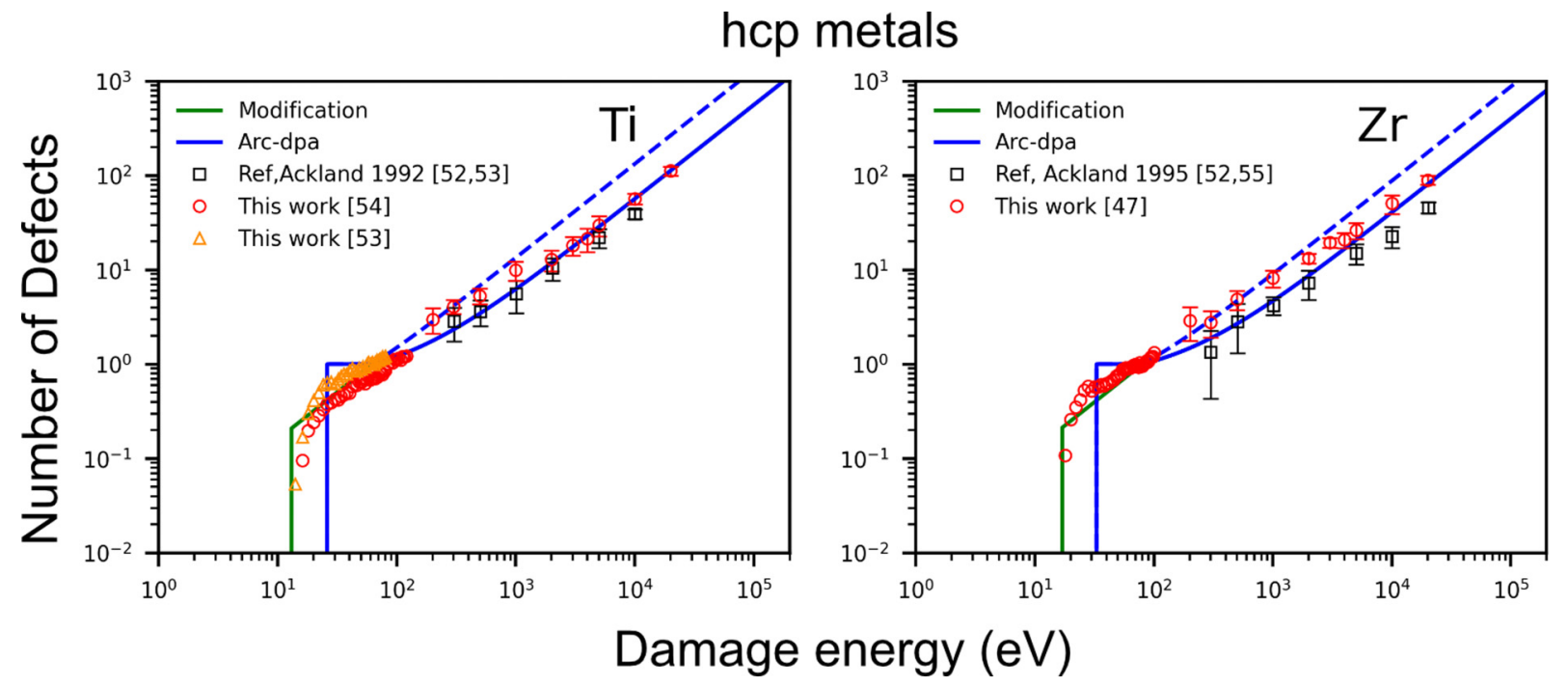

FIG. 4. Damage production in hexagonal close-packed (hcp) metals. Classical molecular dynamics (MD) simulations, as well as the athermal recombination corrected displacements per atom (arc-dpa) model and our low-energy extension are compared. For our own classical MD simulations, the reference numbers are the interatomic potentials used in this work; for the reference damage data using classical MD simulations, we name them as last name of the interatomic potential developer and publication year of the potential for better comparison. The reference numbers for reference classical MD data contain both simulation works and potential development works. Reference data: Ti: Ackland 1992 [52,53]; Zr: Ackland 1995 [52,55]. Interatomic potentials used in this work: Ti [53,54], Zr [47]. $E_{d}^{\mathrm{min}}$ and $E_{d}^{\text {avr }}$ were calculated from classical MD simulations for self-consistency. The error bars correspond to standard deviation.

and second type of TDE surfaces, respectively. Based on both experimental and simulation results, we conclude that TDE surfaces of fcc metals are qualitatively different from those of bcc and hcp metals. This difference causes the nonlinearities in the simulation results. Nevertheless, since the overall agreement between simulation results and the modified model is still good, it should still be very useful for damage production estimation also in fcc metals.

For the high energy damage events $\left(T_{d}>300 \mathrm{eV}\right)$, we also performed many simulations of collision cascades and compared them with other available experimental and simulation results in Figs. 2-4. For bcc and fcc metal materials, $b_{\text {arcdpa }}$ and $c_{\text {ardpa }}$ parameters in Eq. (2) are taken from Refs. [9,26]. However, we note that the $c_{\text {arcdpa }}$ values are not so well fitted for hcp Ti and $\mathrm{Zr}$ in Ref. [26], see the dashed lines for $\mathrm{Ti}$ and $\mathrm{Zr}$ in Fig. 4. Therefore, by following the method in Ref. [26], we refitted $c_{\text {arcdpa }}$ of Ti and Zr. Table I shows the refitted $c_{\text {arcdpa }}$ values for Ti and $\mathrm{Zr}$, while $b_{\text {ardpa }}$ is unchanged. Based on Ref. [26], it is in principle reasonable to assume that $b_{\text {arcdpa }}=-1$ for most materials, including $\mathrm{Ti}$ and $\mathrm{Zr}$. Figures 2-4 demonstrate that the arc-dpa model excellently agrees with bcc, fcc, and hcp metals at high $T_{d}$.

\section{AIMD calculation of minimum TDEs of metals}

Because we note that the minimum TDE carries particular importance for near-threshold events, and we therefore introduced the term $E_{d}^{\min }$ in Eq. (5), we have calculated the minimum TDEs for various metal materials using both AIMD and classical MD. These are listed in Table II in comparison with experimental data. Based on the classical MD results, it is illustrated that, generally, the minimum TDE direction is one of the specific high-symmetry lattice directions, such as $\langle 100\rangle$, $\langle 110\rangle$, and $\langle 111\rangle$, or the global minimum TDE is only $\sim 2 \mathrm{eV}$
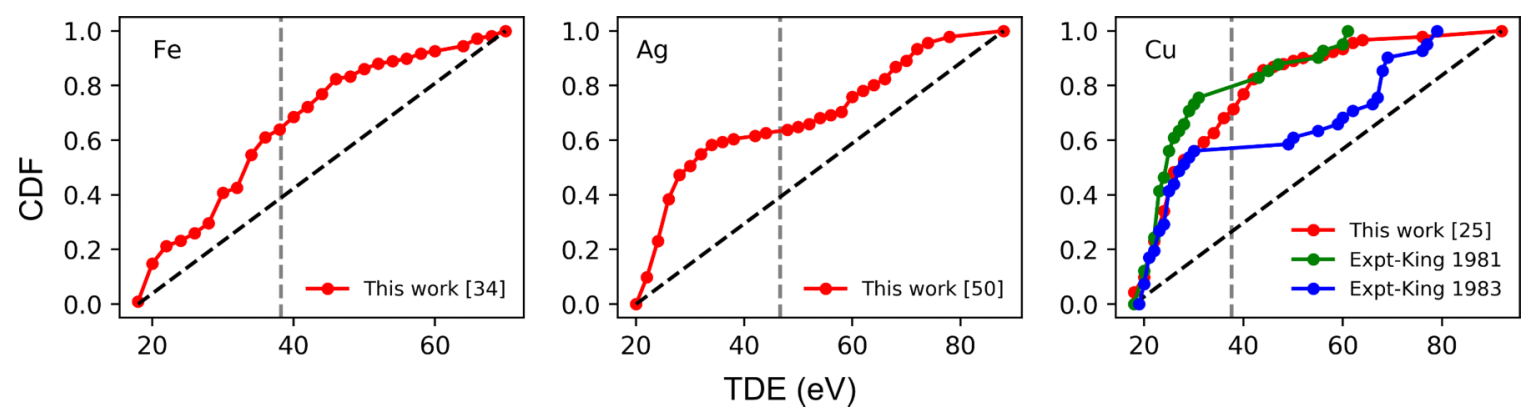

FIG. 5. The cumulative distribution function (CDF) of threshold displacement energy (TDE) distributions in Fe, $\mathrm{Ag}$, and $\mathrm{Cu}$. For our own classical molecular dynamics (MD) simulations, the reference numbers are the interatomic potentials used in this work. The dashed black lines are the CDF of uniformly distributed TDEs, which is the ideal assumption used in the modified model. The dashed gray lines are the average TDE calculated from classical MD simulations. The interatomic potentials are Fe [34], $\mathrm{Ag}$ [50], $\mathrm{Cu}$ [25]. The experimental references are King 1981 [10] and King 1983 [11]. 
TABLE I. Comparison of reference and refitted $c_{\text {arcdpa }}$ for Ti and $\mathrm{Zr}$.

\begin{tabular}{lcc}
\hline \hline$c_{\text {arcdpa }}$ & $\mathrm{Ti}$ & $\mathrm{Zr}$ \\
\hline Reference [26] & 0.83 & 0.70 \\
Refitted & 0.35 & 0.32 \\
\hline \hline
\end{tabular}

lower than that of the lowest high-symmetry direction. Hence, we conclude that AIMD simulations can be a very powerful predictive tool here. As described in Sec. IIC, we only try to find minimum TDEs in several high-symmetry lattice directions but expect these to either be the actual minima or be very near them. The AIMD results are demonstrated in Table II. In general, the AIMD results agree very well with the available experimental results. These results support our assumption that minimum TDEs usually exist in specific high-symmetry directions.

\section{Revising the TDE of fec $\mathrm{Ni}$}

For fcc $\mathrm{Ni}$, we note that the calculated average TDE from classical MD simulations is $64 \mathrm{eV}$ [47] and $82 \mathrm{eV}$ [36], both of which are significantly different from the commonly used literature value of $40 \mathrm{eV}$ from American Society for Testing and Materials (ASTM) [60]. We also note that experiments and AIMD simulations of the minimum values in Table II are significantly lower than those of classical MD simulations.
Ni-based alloys exhibit considerably interesting mechanical properties and good radiation tolerance [61], which make them promising candidates as structural materials in Gen-IV fission reactors. Hence, finding the correct TDE of $\mathrm{Ni}$ is an important step for studying $\mathrm{Ni}$ and $\mathrm{Ni}$-based alloys under irradiation. The TDE of Ni has been determined using both experiments and simulations $[16,56,60,62]$. However, different methods yield significantly different results. The TDE of $\mathrm{Ni}$ thus needs to be more accurately determined. We utilized AIMD calculations to determine the TDEs in three specific lattice directions in $\mathrm{Ni}$ where experimental references exist. The TDEs in these specific lattice directions can be compared with other results to estimate the actual average TDE of Ni. We also used two more interatomic potentials to calculate TDEs of Ni. The results are listed in Table III. The TDEs obtained using different potentials are very different. This is, however, quite expected because the TDEs are significantly influenced by the potential fitting methods applied. While all potentials give different TDEs, they all suggest that the average TDE is much higher than $40 \mathrm{eV}$. This is further supported by AIMD calculations. Even though AIMD simulations present some differences with respect to the experimental results, they still clearly support the general levels and level differences present in the experimental results and, consequently, that the low ASTM value is unreasonable. We conclude that the $40 \mathrm{eV}$ recommendation for Ni from ASTM should be updated in future work and that $\sim 70 \mathrm{eV}$, as indicated

TABLE II. Minimum TDEs of various metals, both experimental and simulation results.

\begin{tabular}{lccccc}
\hline \hline & & & & \multicolumn{2}{c}{ Classical MD } \\
\cline { 3 - 5 } Crystal structure & Metal & Experiment $(\mathrm{eV})$ & AIMD $(\mathrm{eV})$ & Minimum (eV) & Specific directions (eV) \\
\hline bcc & $\mathrm{V}^{\mathrm{a}}$ & $25^{\mathrm{b}}, 30\langle 100\rangle^{\mathrm{c}}$ & $26\langle 100\rangle$ & 12 & $12\langle 100\rangle$ \\
& $\mathrm{Fe}^{\mathrm{d}}$ & $20^{\mathrm{c}}$ & $18^{\mathrm{e}}$ & 18 & $20\langle 100\rangle$ \\
& $\mathrm{Nb}^{\mathrm{f}}$ & $28^{\mathrm{b}}$ & $28\langle 100\rangle$ & 20 & $20\langle 100\rangle$ \\
$\mathrm{fcc}$ & $\mathrm{W}^{\mathrm{g}}$ & $42\langle 100\rangle^{\mathrm{h}}$ & $48\langle 410\rangle^{\mathrm{i}}$ & 36 & $36\langle 100\rangle$ \\
& $\mathrm{Al}^{\mathrm{j}}$ & $16^{\mathrm{b}}$ & $19\langle 100\rangle$ & 14 & $16\langle 100\rangle\langle 110\rangle$ \\
& $\mathrm{Ni}^{\mathrm{k}}$ & $23^{\mathrm{b}}, 22\langle 110\rangle^{\mathrm{c}}$ & $27\langle 100\rangle$ & 38 & $20\langle 110\rangle$ \\
& $\mathrm{Cu}^{\mathrm{l}}$ & $19\langle 110\rangle\langle 100\rangle^{\mathrm{c}}$ & $25\langle 100\rangle$ & 18 & $22\langle 100\rangle$ \\
$\mathrm{hcp}$ & $\mathrm{Ag}^{\mathrm{m}}$ & $23\langle 110\rangle^{\mathrm{c}}$ & $24\langle 100\rangle$ & 20 & $12\langle 0001\rangle$ \\
& $\mathrm{Ti}^{\mathrm{n}}$ & $23\langle 0001\rangle^{\mathrm{o}}$ & $23\langle 0001\rangle$ & 12 & $22\langle 1120\rangle$ \\
\hline \hline
\end{tabular}

\footnotetext{
${ }^{\text {a Reference [30]. }}$

${ }^{b}$ Reference [56].

${ }^{\mathrm{c}}$ Reference [16].

${ }^{\mathrm{d}}$ Reference [34].

${ }^{\mathrm{e}}$ Reference [18].

${ }^{\mathrm{f}}$ Reference [36].

${ }^{\mathrm{g}}$ Reference [40].

${ }^{\mathrm{h}}$ Reference [57].

${ }^{\mathrm{i}}$ Reference [24].

${ }^{\mathrm{j}}$ Reference [44].

${ }^{\mathrm{k}}$ Reference [47].

${ }^{\mathrm{l}}$ Reference [25].

${ }^{\mathrm{m}}$ Reference [50].

${ }^{\mathrm{n}}$ Reference [54].

${ }^{\circ}$ Reference [58].

${ }^{\mathrm{P}}$ Reference [47].

${ }^{\mathrm{q}}$ Reference [59].
} 
TABLE III. Average TDE and TDEs in specific directions for Ni. For MD simulations, the potentials used are named as last name of first author and publication year.

\begin{tabular}{|c|c|c|c|c|c|c|c|}
\hline & \multicolumn{2}{|c|}{ Reference (eV) } & \multicolumn{5}{|c|}{ This paper $(\mathrm{eV})$} \\
\hline & ASTM $^{\mathrm{a}}$ & Experiment & AIMD & Wilson $2015^{\mathrm{b}}$ & Zhang $2016^{c}$ & Ko $2015^{\text {d }}$ & Bonny $2013^{\mathrm{e}}$ \\
\hline$\langle 100\rangle$ & & $38^{\mathrm{f}}$ & 27 & 46 & 50 & 28 & 52 \\
\hline$\langle 110\rangle$ & & $21^{\mathrm{f}}$ & 30 & 40 & 44 & 32 & 52 \\
\hline$\langle 111\rangle$ & & $>60^{\mathrm{f}}$ & 70 & 70 & 80 & 40 & 80 \\
\hline$E_{d}^{\mathrm{avr}}$ & 40 & $69^{\mathrm{g}}$ & & 65 & 82 & 49 & 100 \\
\hline
\end{tabular}

${ }^{\text {a Reference [60]. }}$

${ }^{\mathrm{b}}$ Reference [47].

${ }^{\mathrm{c}}$ Reference [36].

${ }^{\mathrm{d}}$ Reference [63].

${ }^{\text {e}}$ Reference [64].

${ }^{\mathrm{f}}$ Reference [16].

${ }^{\mathrm{g}}$ Reference [56].

by experiments, seems to be a more consistent and reasonable value.

\section{CONCLUSIONS}

In this paper, we modified the arc-dpa model with a lowenergy extension to make it suitable for near TDE primary damage events and thus provide a simple analytical damage model valid for all energy ranges. Classical MD simulations were used to validate the modified model. It is shown here that these provide predictive data that can be used generally for a wide implementation of the model. In general, the modified model agrees very well with simulation results on metallic materials. For fcc metals, the TDE surfaces are qualitatively different from bcc and hcp metals, which is the reason for the larger deviation between the model and simulation results. We also used AIMD simulations to determine the minimum TDEs for metallic materials in a few high-symmetry directions. The AIMD results are in excellent agreement with experimental results. This means that AIMD can be used to calculate the minimum TDEs of metallic materials with reasonable computational cost and very good accuracy. In addition, the average TDE of $\mathrm{Ni}$ is estimated using both MD and AIMD. By comparing the simulation data with experimental results, it is indicated that the commonly used literature TDE of $\mathrm{Ni}$ $(40 \mathrm{eV})$ is incorrect, and the true average TDE of $\mathrm{Ni}$ is closer to $70 \mathrm{eV}$.

\section{ACKNOWLEDGMENTS}

The financial support by the China Scholarship Council (No. 201807930008), Svensk Kärnbränslehantering AB and the EUROfusion Consortium through the Euratom research and training programme 2019-2020 under Grant Agreement No. 633053 is acknowledged. The views and opinions expressed herein do not necessarily reflect those of the European Commission. The computational resources were provided by the Swedish National Infrastructure for Computing (SNIC) and by the CINECA HPC center in Italy. The authors gratefully thank Elin Toijer, Luca Messina, and Zhongwen Chang for inspiration as well as Kai Nordlund at University of Helsinki for fruitful discussions. This paper contributes to the Joint Program on Nuclear Materials (JPNM) of the European Energy Research Alliance (EERA).
[1] D. Schulz-Ertner and H. Tsujii, Particle radiation therapy using proton and heavier ion beams, J. Clin. Oncol. 25, 953 (2007).

[2] CMS Collaboration et al., Combined results of searches for the standard model Higgs boson in pp collisions at $\sqrt{s}=7 \mathrm{TeV}$, Phys. Lett. B 710, 26 (2012).

[3] E. Chason, S. T. Picraux, J. M. Poate, J. O. Borland, M. I. Current, T. Diaz de la Rubia, D. J. Eaglesham, O. W. Holland, M. E. Law, C. W. Magee, J. W. Mayer, J. Melngailis, and A. F. Tasch, Ion beams in silicon processing and characterization, J. Appl. Phys. 81, 6513 (1997).

[4] K. Nordlund, S. J. Zinkle, A. E. Sand, F. Granberg, R. S. Averback, R. E. Stoller, T. Suzudo, L. Malerba, F. Banhart, W. J. Weber, F. Willaime, S. L. Dudarev, and D. Simeone, Primary radiation damage: a review of current understanding and models, J. Nucl. Mater. 512, 450 (2018).

[5] S. J. Zinkle and G. S. Was, Materials challenges in nuclear energy, Acta Mater. 61, 735 (2013).
[6] G. H. Kinchin and R. S. Pease, The displacement of atoms in solids by radiation, Rep. Prog. Phys. 18, 1 (1955).

[7] M. J. Norgett, M. T. Robinson, and I. M. Torrens, A proposed method of calculating displacement dose rates, Nucl. Eng. Des. 33, 50 (1975).

[8] R. S. Averback and T. D. De La Rubia, Displacement damage in irradiated metals and semiconductors, Solid State Phys. 51, 281 (1998).

[9] K. Nordlund, S. J. Zinkle, A. E. Sand, F. Granberg, R. S. Averback, R. Stoller, T. Suzudo, L. Malerba, F. Banhart, W. J. Weber, F. Willaime, S. L. Dudarev, and D. Simeone, Improving atomic displacement and replacement calculations with physically realistic damage models, Nat. Commun. 9, 1084 (2018).

[10] W. E. King, K. L. Merkle, and M. Meshii, Determination of the threshold-energy surface for copper using in-situ electrical-resistivity measurements in the high-voltage electron microscope, Phys. Rev. B 23, 6319 (1981). 
[11] W. E. King, K. L. Merkle, and M. Meshii, Threshold energy surface and Frenkel pair resistivity for $\mathrm{Cu}$, J. Nucl. Mater. 117, 12 (1983).

[12] S. J. Zinkle and B. N. Singh, Analysis of displacement damage and defect production under cascade damage conditions, J. Nucl. Mater. 199, 173 (1993).

[13] K. Nordlund, J. Wallenius, and L. Malerba, Molecular dynamics simulations of threshold displacement energies in $\mathrm{Fe}$, Nucl. Instrum. Meth. B 246, 322 (2006).

[14] A. H. Compton, A quantum theory of the scattering of x-rays by light elements, Phys. Rev. 21, 483 (1923).

[15] G. S. WAS, Fundamentals of Radiation Materials Science (Springer, New York, 2017).

[16] P. Vajda, Anisotropy of electron radiation damage in metal crystals, Rev. Mod. Phys. 49, 481 (1977).

[17] S. Plimpton, Fast parallel algorithms for short-range molecular dynamics, J. Comput. Phys. 117, 1 (1995).

[18] P. Olsson, C. S. Becquart, and C. Domain, Ab initio threshold displacement energies in iron, Mater. Res. Lett. 4, 219 (2016).

[19] A. Stukowski, Visualization and analysis of atomistic simulation data with OVITO - the open visualization tool, Model. Simul. Mater. Sci. Eng. 18, 015012 (2010).

[20] E. Wigner and F. Seitz, On the constitution of metallic sodium, Phys. Rev. 43, 804 (1933).

[21] G. Kresse and J. Furthmüller, Efficient iterative schemes for ab initio total-energy calculations using a plane-wave basis set, Phys. Rev. B 54, 11169 (1996).

[22] P. E. Blöchl, Projector augmented-wave method, Phys. Rev. B 50, 17953 (1994).

[23] J. P. Perdew, K. Burke, and M. Ernzerhof, Generalized Gradient Approximation Made Simple, Phys. Rev. Lett. 77, 3865 (1996).

[24] A. De Backer, A. Sand, C. J. Ortiz, C. Domain, P. Olsson, E. Berthod, and C. S. Becquart, Primary damage in tungsten using the binary collision approximation, molecular dynamic simulations and the density functional theory, Phys. Scr. 2016, 014018 (2016).

[25] V. Borovikov, M. I. Mendelev, and A. H. King, Effects of stable and unstable stacking fault energy on dislocation nucleation in nano-crystalline metals, Model. Simul. Mater. Sci. Eng. 24, 085017 (2016).

[26] A. Y. Konobeyev, U. Fischer, Y. A. Korovin, and S. P. Simakov, Evaluation of effective threshold displacement energies and other data required for the calculation of advanced atomic displacement cross-sections, Nucl. Energy Technol. 3, 169 (2017).

[27] A. Akkerman and J. Barak, New partition factor calculations for evaluating the damage of low energy ions in silicon, IEEE Trans. Nucl. Sci. 53, 3667 (2006).

[28] K. Morishita, T. Diaz De, and La Rubia, A molecular dynamics simulation study of displacement cascades in vanadium, J. Nucl. Mater. 271-272, 35 (1999).

[29] R. A. Johnson and D. J. Oh, Analytic embedded atom method model for bcc metals, J. Mater. Res. 4, 1195 (1989).

[30] M. I. Mendelev, S. Han, W. J. Son, G. J. Ackland, and D. J. Srolovitz, Simulation of the interaction between Fe impurities and point defects in V, Phys. Rev. B 76, 214105 (2007).

[31] R. E. Stoller, Primary radiation damage formation, Compr. Nucl. Mater. 1, 293 (2012).

[32] A. F. Calder and D. J. Bacon, A molecular dynamics study of displacement cascades in $\alpha$-iron, J. Nucl. Mater. 207, 25 (1993).

[33] L. Malerba, M. C. Marinica, N. Anento, C. Björkas, H. Nguyen,
C. Domain, F. Djurabekova, P. Olsson, K. Nordlund, A. Serra, D. Terentyev, F. Willaime, and C. S. Becquart, Comparison of empirical interatomic potentials for iron applied to radiation damage studies, J. Nucl. Mater. 406, 19 (2010).

[34] G. J. Ackland, M. I. Mendelev, D. J. Srolovitz, S. Han, and A. V. Barashev, Development of an interatomic potential for phosphorus impurities in $\alpha$-iron, J. Phys. Condens. Matter 16, S2629 (2004).

[35] D.-Y. Lin, H. Song, and X. D. Hui, Molecular dynamics simulation of threshold displacement energy and primary damage state in niobium, arXiv:1702.03598v2 (2017).

[36] Y. Zhang, R. Ashcraft, M. I. Mendelev, C. Z. Wang, and K. F. Kelton, Experimental and molecular dynamics simulation study of structure of liquid and amorphous $\mathrm{Ni}_{62} \mathrm{Nb}_{38}$ Alloy, J. Chem. Phys. 145, 204505 (2016).

[37] M. R. Fellinger, H. Park, and J. W. Wilkins, Force-matched embedded-atom method potential for niobium, Phys. Rev. B 81 , 144119 (2010).

[38] G. J. Ackland and R. Thetford, An improved N-body semiempirical model for body-centred cubic transition metals, Philos. Mag. A 56, 15 (1987).

[39] C. Björkas, K. Nordlund, and S. Dudarev, Modelling radiation effects using the $a b$-initio based tungsten and vanadium potentials, Nucl. Instrum. Meth. B 267, 3204 (2009).

[40] M. Marinica, L. Ventelon, M. R. Gilbert, L. Proville, S. L. Dudarev, J. Marian, G. Bencteux, and F. Willaime, Interatomic potentials for modelling radiation defects and dislocations in tungsten, J. Phys. Condens. Matter 25, 395502 (2013).

[41] S. Han, L. A. Zepeda-Ruiz, G. J. Ackland, R. Car, and D. J. Srolovitz, Interatomic potential for vanadium suitable for radiation damage simulations, J. Appl. Phys. 93, 3328 (2003).

[42] A. Almazouzi, M. J. Caturla, M. Alurralde, T. Diaz De La Rubia, and M. Victoria, Defect production and damage evolution in $\mathrm{Al}$ : a molecular dynamics and Monte Carlo computer simulation, Nucl. Instrum. Meth. B 153, 105 (1999).

[43] F. Ercolesi and J. B. Adams, Interatomic potentials from firstprinciples calculations: the force-matching method, EPL 26, 583 (1994)

[44] M. I. Mendelev, F. Zhang, Z. Ye, Y. Sun, M. C. Nguyen, S. R. Wilson, C. Z. Wang, and K. M. Ho, Development of interatomic potentials appropriate for simulation of devitrification of $\mathrm{Al}_{90} \mathrm{Sm}_{10}$ alloy, Model. Simul. Mater. Sci. Eng. 23, 045013 (2015).

[45] A. Almazouzi, M. J. Caturla, T. D. de la Rubia, and M. Victoria, Annealing kinetics of single displacement cascades in $\mathrm{Ni}$ : an atomic scale computer simulation, MRS Proc. 540, 685 (1998).

[46] F. Cleri and V. Rosato, Tight-binding potentials for transition metals and alloys, Phys. Rev. B 48, 22 (1993).

[47] S. R. Wilson and M. I. Mendelev, Anisotropy of the solid-liquid interface properties of the Ni-Zr B33 phase from molecular dynamics simulation, Philos. Mag. 95, 224 (2015).

[48] M. J. Sabochick and N. Q. Lam, Radiation-induced amorphization of ordered intermetallic compounds $\mathrm{CuTi}, \mathrm{CuTi}_{2}$, and $\mathrm{Cu}_{4} \mathrm{Ti}_{3}$ : a molecular-dynamics study, Phys. Rev. B 43, 5243 (1991).

[49] K. L. Merkle, R. S. Averback, and R. Benedek, Energy Dependence of Defect Production in Displacement Cascades in Silver, Phys. Rev. Lett. 38, 424 (1977).

[50] Z. Pan, V. Borovikov, M. I. Mendelev, and F. Sansoz, Development of a semi-empirical potential for simulation of $\mathrm{Ni}$ solute 
segregation into grain boundaries in Ag, Model. Simul. Mater. Sci. Eng. 26, 075004 (2018).

[51] P. L. Williams, Y. Mishin, and J. C. Hamilton, An embeddedatom potential for the $\mathrm{Cu}-\mathrm{Ag}$ system, Model. Simul. Mater. Sci. Eng. 14, 817 (2006).

[52] S. J. Wooding, L. M. Howe, F. Gao, A. F. Calder, and D. J. Bacon, A molecular dynamics study of high-energy displacement cascades in $\alpha$-zirconium, J. Nucl. Mater. 254, 191 (1998).

[53] G. J. Ackland, Theoretical study of titanium surfaces and defects with a new many-body potential, Philos. Mag. A 66, 917 (1992).

[54] R. R. Zope and Y. Mishin, Interatomic potentials for atomistic simulations of the Ti-Al system, Phys. Rev. B 68, 024102 (2003).

[55] G. J. Ackland, S. J. Wooding, and D. J. Bacon, Defect, surface and displacement-threshold properties of $\alpha$-zirconium simulated with a many-body potential, Philos. Mag. A 71, 553 (1995).

[56] P. Jung, Average atomic-displacement energies of cubic metals, Phys. Rev. B 23, 664 (1981).

[57] F. Maury, M. Biget, P. Vajda, A. Lucasson, and P. Lucasson, Frenkel pair creation and stage I recovery in W crystals irradiated near threshold, Radiat. Eff. 38, 53 (1978).

[58] A. S. A. Karim, M. E. Whitehead, M. H. Loretto, and R. E. Smallman, Electron radiation damage in hcp metals-I. The determination of the threshold displacement energy in $\mathrm{Zn}, \mathrm{Cd}$, Mg and Ti, Acta Metall. 26, 975 (1978).

[59] M. Biget, F. Maury, P. Vajda, A. Lucasson, and P. Lucasson, Production and mutual annihilation of Frenkel pairs in low temperature irradiated zirconium, Radiat. Eff. 7, 223 (1971).

[60] ASTM E521, Standard practice for investigating the effects of neutron radiation damage, in Annual Book of ASTM Standards, Vol. 12 (ASTM International, West Conshohocken, PA, 2009).

[61] C. Lu, L. Niu, N. Chen, K. Jin, T. Yang, P. Xiu, Y. Zhang, F. Gao, H. Bei, S. Shi, M.-R. He, I. M. Robertson, W. J. Weber, and L. Wang, Enhancing radiation tolerance by controlling defect mobility and migration pathways in multicomponent single-phase alloys, Nat. Commun. 7, 13564 (2016).

[62] L. K. Béland, C. Lu, Y. N. Osetskiy, G. D. Samolyuk, A. Caro, L. Wang, and R. E. Stoller, Features of primary damage by high energy displacement cascades in concentrated Ni-based alloys, J. Appl. Phys. 119, 085901 (2016).

[63] W.-S. Ko, B. Grabowski, and J. Neugebauer, Development and application of a Ni-Ti interatomic potential with high predictive accuracy of the martensitic phase transition, Phys. Rev. B 92, 134107 (2015).

[64] G. Bonny, N. Castin, and D. Terentyev, Interatomic potential for studying ageing under irradiation in stainless steels: the FeNiCr model alloy, Model. Simul. Mater. Sci. Eng. 21, 085004 (2013). 